\title{
Parameterized Reduced Order Models with Guaranteed Passivity using Matrix Interpolation
}

\author{
Elizabeth Rita Samuel, Francesco Ferranti, Luc Knockaert and Tom Dhaene \\ Dept. of Information Technology (INTEC) \\ Ghent University - IBBT,Gaston Crommenlaan 8 Bus 201, B-9050 Gent, Belgium \\ Email: elizabeth.ritasamuel@ugent.be,francesco.ferranti@ugent.be, luc.knockaert@ugent.be, tom.dhaene@ugent.be
}

\begin{abstract}
We present a novel parameterized model order reduction method based on matrix interpolation. The design space is sampled over an estimation grid and for each estimation point a Krylov subspace is computed. A common projection matrix is generated by the truncation of the singular values of the merged Krylov subspaces of all estimation points from the design space. The reduced matrices are then interpolated using positive interpolation schemes to build guaranteed passive parameterized reduced order models. The technique is validated by means of a pertinent numerical simulation.
\end{abstract}

\section{Introduction}

Electromagnetic (EM) methods [1,2] have become an indispensable analysis and design tool for a variety of complex highspeed systems. However, a drawback of EM methods is that they usually generate very large systems of equations. The aim to decrease the simulation time in analysis and design led to the development of model order reduction (MOR) techniques which ultimately have to retain accuracy and physical system properties [3-6].

Non-parametric MOR techniques perform model reduction only with respect to frequency. However, in the circuit synthesis of large-scale digital or analog applications, it is also important to predict the response of the circuit as a function of environmental effects, manufacturing variations, and fluctuations in the critical dimensions. These design activities call for parameterized model order reduction (PMOR) methods that can reduce large systems of equations with respect to both frequency and also other design parameters of the circuit, such as geometrical layout or substrate characteristics.

Different PMOR methods have developed in recent years. The multiparameter moment-matching methods presented in [7, 8] use a subspace projection approach, but the resulting reduced order models (ROMs) usually suffer from oversize. The technique presented in [9] combines traditional passivitypreserving model order reduction methods and positive interpolation schemes that are applied to an input-output system level. A PMOR method based on a parameterization process of matrices generated by EM methods and projection subspaces is proposed in [10]. The overall passivity of the parameterized ROMs is guaranteed over the design space of interest in $[9,10]$. A matrix interpolation based technique has been proposed in [11] which avoids the oversize problem of multiparameter moment matching based algorithms, but in [11] the reduced system matrices needed for interpolation must have the same order and must be post processed for reprojection onto a common subspace. Unfortunately, passivity is not guaranteed.

In this paper, we propose a PMOR method that remediates the flaws of [11] by: 1) using a common projection matrix for the entire design space and 2) using passivity preserving parameterization schemes. The truncation of the singular values of the merged Krylov subspaces computed from the models at the estimation points of the design space generates a common projection matrix. Next, the reduced system matrices are interpolated using positive interpolation schemes in order to obtain a passive parameterized reduced model. The Krylov subspaces can be found by means of Krylov-based MOR methods. In this paper we use the Laguerre-SVD technique [6]. A flowchart describing the different steps of the proposed technique is shown in Fig.1.

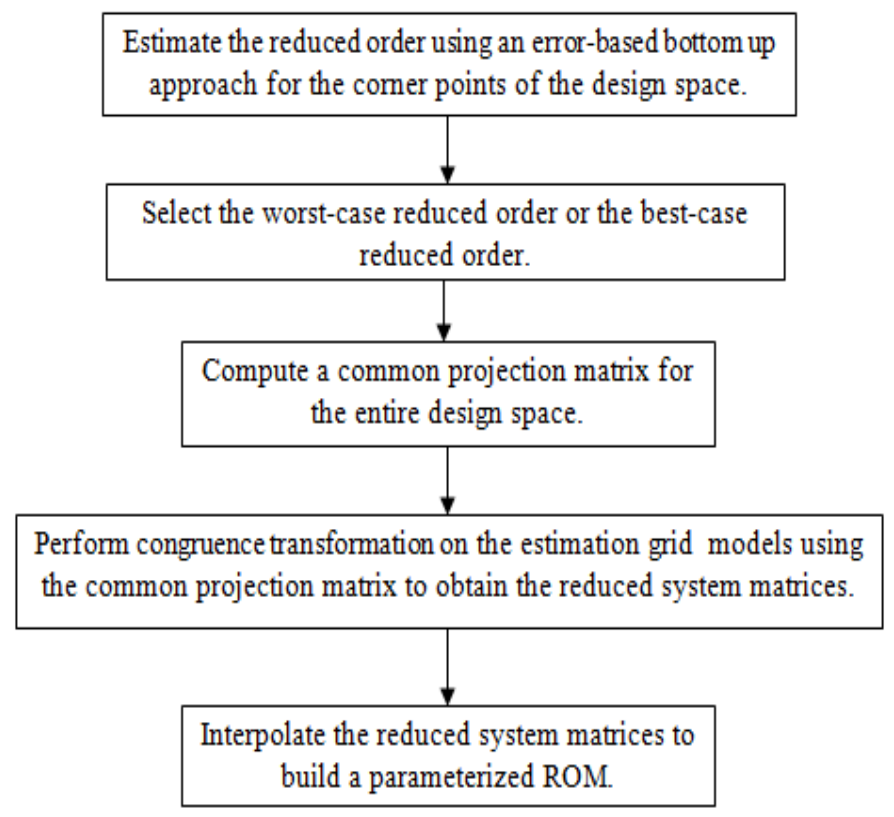

Figure 1: Flowchart of the proposed technique.

This paper is organized as follows. Section 2 describes the determination of the reduced order over the estimation grid. The generation of the common projection matrix is described in Section 3. Section 4 discusses the multivariate interpolation of the reduced system matrices and the preservation of passivity for the generation of a passive parameterized ROM. A pertinent numerical example validates the proposed technique in Section 5 .

\section{Determination of the reduced order}

The determination of the reduced order is important for an efficient construction of parameterized ROMs. The corner points of the design space are essential for the reduced order estimation. Note that the design space does not include frequency 
[10]. Two design space grids are used in the modeling process, namely an estimation and a validation grid. The parameterized ROMs are estimated using the estimation grid and validated over the validation grid.

First, the reduced order is determined at the corner points of the design space using an error-based bottom-up approach. The weighted rms error defined as [10],

$$
\begin{array}{r}
\operatorname{Err}\left(\mathbf{Y}_{1}(s), \mathbf{Y}_{2}(s)\right)=\sqrt{\frac{\sum_{k=1}^{K_{s}} \sum_{i=1}^{P_{i n}} \sum_{j=1}^{P_{\text {out }}} \frac{\left|Y_{1,(i j)}\left(s_{k}\right)-Y_{2,(i j)}\left(s_{k}\right)\right|^{2}}{W_{(i j)}\left(s_{k}\right)}}{P_{\text {in }} P_{\text {out }} K_{s}}} \\
W_{(i j)}\left(s_{k}\right)=\left|Y_{2,(i j)}\left(s_{k}\right)\right|^{2}
\end{array}
$$

is used for computing the reduced order in the bottom-up approach. In (1) $K_{s}, P_{\text {in }}$ and $P_{\text {out }}$ are the number of frequency samples, input and output ports of the system, respectively.

Next, the reduced order for the other estimation points can be chosen in two ways:

1. worst-case reduced order: the highest estimated reduced order at the corner points is extended to the entire estimation grid. This approach can guarantee an accurate reduction over the design space.

2. best-case reduced order: the lowest estimated reduced order is extended to the entire estimation grid. This approach can guarantee more compact models with respect to the previous one, but the reduced order may have to be increased for some design space regions to guarantee the desired accuracy.

\section{Common projection matrix computation}

Considering a parameterized dynamical system with design parameters $\mathbf{g}=\left(g^{(1)}, \ldots, g^{(N)}\right)$, the model is expressed as the following descriptor state space form:

$$
\begin{aligned}
\mathbf{C}(\mathbf{g}) \frac{d \mathbf{x}(t, \mathbf{g})}{d t} & =-\mathbf{G}(\mathbf{g}) \mathbf{x}(t, \mathbf{g})+\mathbf{B u}(t) \\
\mathbf{y}(t, \mathbf{g}) & =\mathbf{L}^{\prime} \mathbf{x}(t, \mathbf{g})+\mathbf{D u}(t)
\end{aligned}
$$

All the projection matrices will have the same dimension for the worst-case reduced order scenario, while they may have different dimensions for the best-case reduced order scenario. For each estimation point in the design space, a Krylov-based MOR method is applied to the corresponding system, yielding a set of projection matrices. In this paper the Laguerre-SVD [6] method is used.

The projection matrices are computed over the estimation grid and merged by column stacking

$$
\mathbf{P}_{\text {union }}=\left[P_{1}, P_{2}, \ldots . . P_{\text {Est }}\right]
$$

where $E s t_{p t}$ is the number of estimation points. The order of $\mathbf{P}_{\text {union }}$ is $n \times p$ with $p=\left(q_{1}+q_{2} \ldots+q_{\text {Est } p t}\right)$, where $q_{i}$ is the reduced order for each estimation point $i$ as determined in Section 2. Then, the economy size singular value decomposition [12] is computed for the union of the projection matrices.

$$
\mathbf{U} \Sigma \mathbf{V}^{\prime}=\operatorname{svd}\left(\mathbf{P}_{\text {union }}\right)
$$

A common reduced order $r$ for a cell is defined based on the first $r$ significant singular values by setting a threshold to the ratio of the singular values with respect to the largest singular value $\sigma_{\max }$ :

$$
\frac{\sigma_{i}}{\sigma_{\max }} \geq \text { threshold }_{\sigma}, i=1,2, \ldots \ldots \ldots, r
$$

A common projection matrix $\mathbf{Q}_{\text {comm }}$ is obtained for the entire design space by QR orthonormalization on $\mathbf{P}_{\text {comm }}$

$$
\begin{aligned}
\mathbf{P}_{\text {comm }} & =\mathbf{U}_{r} \Sigma_{r} \mathbf{V}_{r}^{\prime} \\
{\left[\mathbf{Q}_{\text {comm }}, \mathbf{R}\right] } & =q r\left(P_{\text {comm }}\right)
\end{aligned}
$$

The congruence transformation using the common projection matrix $\mathbf{Q}_{\text {comm }}$ on the original model gives the reduced system matrices for the estimation points in the design space.

Regarding the state-space equations of the system under study, we assume that a topologically fixed discretization mesh is used and is independent of the specific design parameter values [10]. The sizes of the system matrices are preserved as well as the numbering of the mesh nodes and mesh edges. When the shape parameters are modified the mesh is only locally stretched or shrunk. The matrices $\mathbf{B}, \mathbf{L}^{\prime}$ are uniquely determined by the circuit topology and therefore remains constant, while the matrices $\mathbf{C}$ and $\mathbf{G}$ are defined as functions of the design parameters. Starting from a set of models in the estimation design space (generated with respect to a common space) using common projection matrices, it is straightforward to prove that all the reduced system matrices in the estimation grid are in the same subspace and hence can be interpolated.

\section{Parameterized Model Order Reduction}

Once the reduced system matrices are obtained for the estimation grid, the parameterized ROM is build by using positive interpolation schemes.

Multivariate interpolation can be realized by means of tensor product [13] or tessellation methods [14]. Any interpolation scheme in the class of positive interpolation operators [9] can be used, e.g., multilinear, and simplicial methods [15], in order to preserve overall passivity.

For multilinear interpolation, each interpolated matrix $\mathbf{J}\left(g^{(1)}, \ldots, g^{(N)}\right)$, can be written as

$$
\begin{array}{r}
\mathbf{J}\left(g^{(1)}, \ldots, g^{(N)}\right)=\quad \sum_{k_{1}=1}^{K_{1}} \cdots \sum_{k_{N}=1}^{K_{N}} \mathbf{J}_{\left(g_{k_{1}}^{(1)}, \ldots, g_{k_{N}}^{(N)}\right)} \\
l_{k_{1}}\left(g^{(1)}\right) \cdots l_{k_{N}}\left(g^{(N)}\right)
\end{array}
$$

where $K_{1}$ is the number of estimation points and $l_{k_{i}}\left(g^{(i)}\right)$ are piecewise linear interpolation kernels. The interpolation kernel satisfies the following constraints

$$
\begin{aligned}
0 \leq \quad l_{k_{i}}\left(g^{(i)}\right) & \leq 1 \\
l_{k_{i}}\left(g^{(i)}\right) & =\delta_{k_{i}, i} \\
\sum_{k=1}^{K_{1}} l_{k_{i}}\left(g^{(i)}\right) & =1
\end{aligned}
$$

Since the interpolation kernel depends on the design space, interpolating systems, matrices or scalars does not make any difference. 
Stability and passivity must be guaranteed when transient analysis is to be performed. It is known that, while a passive system is also stable, the reverse is not necessarily true [16]. Hence the passivity requirement is crucial when the model is to be utilized in a time-domain simulator with drivers and receivers.

When the original models are in the MNA form (2) and if the following conditions are satisfied:

$$
\begin{gathered}
\mathbf{C}=\mathbf{C}^{\prime} \geq 0 \\
\mathbf{G}+\mathbf{G}^{\prime} \geq 0 \\
\mathbf{B}=\mathbf{L}
\end{gathered}
$$

the passivity of the transfer function $\mathbf{Y}(s)=\mathbf{L}^{\prime}(s \mathbf{C}+\mathbf{G})^{-1} \mathbf{B}$ is guaranteed [17]. For this specific descriptor format, the proposed PMOR method guarantees the passivity of the ROMs over the estimation grid using the Laguerre-SVD method (or PRIMA method) by congruence transformation using the common projection matrix $\mathbf{Q}_{\text {comm }}$

$$
\begin{gathered}
\mathbf{C}_{r}(\mathbf{g})=\mathbf{Q}_{\text {comm }}{ }^{\prime} \mathbf{C}(\mathbf{g}) \mathbf{Q}_{\text {comm }} \geq 0 \\
\mathbf{G}_{r}(\mathbf{g})=\mathbf{Q}_{\text {comm }}{ }^{\prime} \mathbf{G}(\mathbf{g}) \mathbf{Q}_{\text {comm }} \geq 0 \\
\mathbf{B}_{r}(\mathbf{g})=\mathbf{Q}_{\text {comm }} \mathbf{B}(\mathbf{g}) \\
\mathbf{L}_{r}(\mathbf{g})=\mathbf{Q}_{\text {comm }^{\prime}} \mathbf{L}(\mathbf{g})
\end{gathered}
$$

Since any nonnegative linear combination of positive semidefinite matrices is a positive semidefinite matrix, stability and passivity are preserved over the entire design space if positive interpolation operators are used.

\section{Numerical examples}

A coupled microstrip structure is modeled as described in [6]. The cross section is shown in Fig.2. The conductors have width

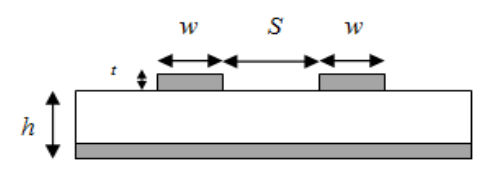

Figure 2: Two coupled microstrip line.

$w=100 \mu \mathrm{m}$ and thickness $t=50 \mu \mathrm{m}$. The length $L$ is considered as a variable parameter in addition to frequency. Their corresponding ranges are shown in Table 1.

\section{Table 1: PARAMETERS OF THE COUPLED LINE}

\begin{tabular}{|l|l|l|}
\hline Parameter & Min & Max \\
\hline Frequency $($ freq) & $1 \mathrm{kHz}$ & $4 \mathrm{GHz}$ \\
Length $(L)$ & $2 \mathrm{~cm}$ & $6 \mathrm{~cm}$ \\
\hline
\end{tabular}

The $\mathbf{C}, \mathbf{G}, \mathbf{B}, \mathbf{L}$ matrices are obtained for 5 values of the length $L$. The order of the original systems is equal to 2002. The reduced order is determined for $L=\{2,6\} \mathrm{cm}$ and it is found to be 26 and 28. The best-case reduced order scenario is selected with an error threshold of 0.01 . The design space is divided into grids, $L=\{2,4,6\} \mathrm{cm}$ is the estimation set and $L=\{3,5\} \mathrm{cm}$ is the validation set. The Krylov subspaces are found using the Laguerre-SVD algorithm for the estimation points. Next, a common projection matrix is computed. If the union of the projection matrices had been used for the congruence transformation, then the reduced order would have been 85 . Due to the truncation of the singular values of the reduced matrices (threshold 0.01 ) the dimension of the common projection matrix is 42 as indicated in Fig. 3. Fig. 4 shows the magnitude

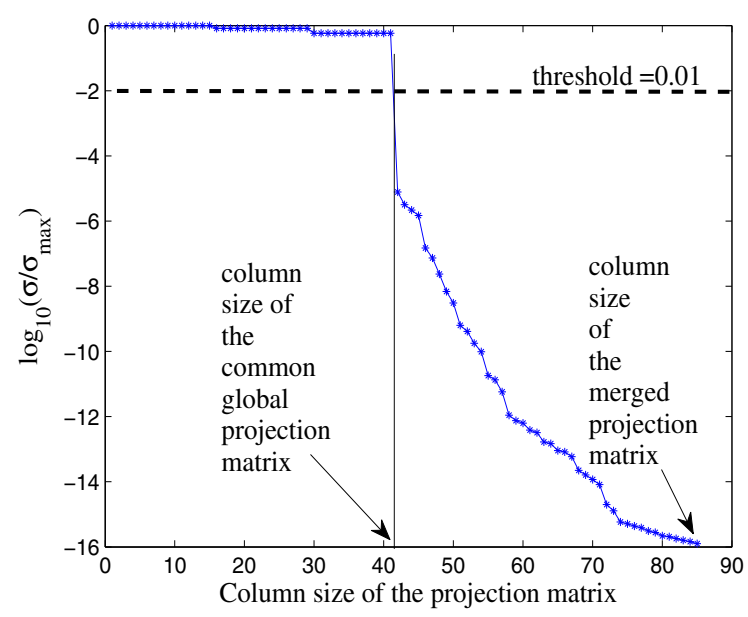

Figure 3: Singular value of the projection matrix.

of the bivariate ROM using linear interpolation. Fig. 5 compares the magnitude of $\mathbf{Y}_{12}(s, L)$ and its model for the validation points $L=\{3,5\} \mathrm{cm}$. The weighted RMS error (1) for all values of $L$ is 0.0196 .

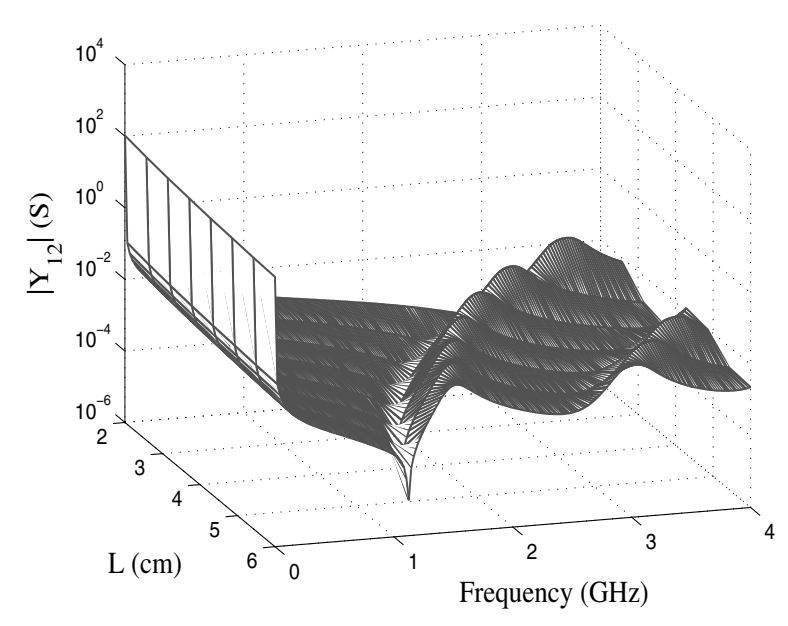

Figure 4: Magnitude of the bivariate reduced model $\mathbf{Y}_{12}(s, L)$.

It is seen that the parameterized ROM captures very accurately the behavior of the system while guaranteeing passivity over the entire design space. 


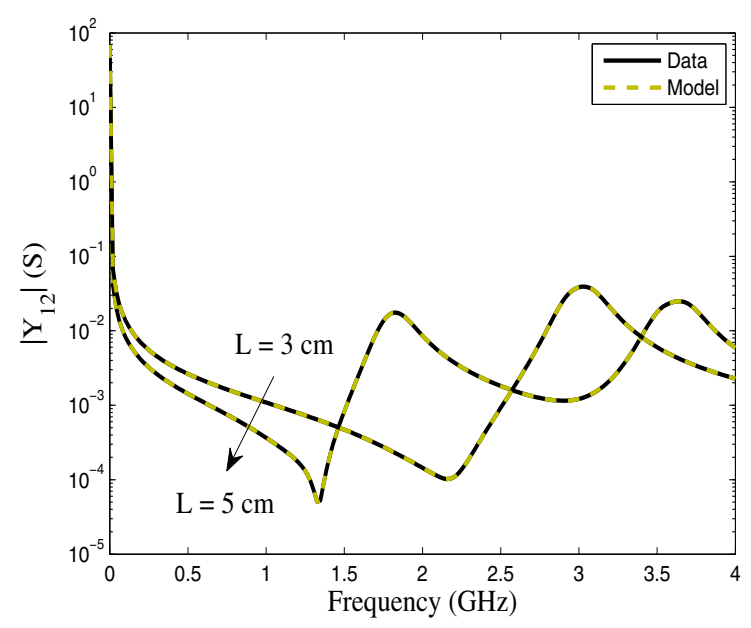

Figure 5: Magnitude of the bivariate reduced model $\mathbf{Y}_{12}(s, L)$ for $L=\{3,5\} \mathrm{cm}$ using a common projection matrix.

\section{Conclusions}

We have presented a novel PMOR method based on matrix interpolation. A common projection matrix is used to build the parameterized model. After the determination of the reduced order, a common projection matrix is generated by the truncation of the singular values of the merged Krylov subspaces of the estimation grid. Next, the reduced system matrices are interpolated using positive interpolation schemes. Overall stability and passivity are guaranteed. Pertinent numerical results confirm the accuracy of the parameterized ROMs.

\section{Acknowledgments}

This work was supported by the Research Foundation Flanders (FWO).

\section{References}

[1] A. Ruehli, "Equivalent circuit models for threedimensional multiconductor systems," IEEE Transactions on Microwave Theory and Techniques, vol. 22, no. 3, pp. 216 - 221, Mar. 1974.

[2] J. M. Jin, The Finite Element Method in Electromagnetics, 2nd ed. NewYork: Wiley, 2001.

[3] K. Gallivan, E. Grimme, and V. P. Dooren, "Asymptotic waveform evaluation via a Lanczos method," Applied Mathematics Letter, vol. 7, no. 5, pp. 75-80, Sept. 1994.

[4] P. Feldmann and W. R. Freund, "Efficient linear circuit analysis by Padè approximation via the Lanczos process," IEEE Transactions on Computer-Aided Design of Integrated Circuits and Systems, vol. 14, no. 5, pp. 639-649, May 1995.

[5] A. Odabasioglu, M. Celik, and L. Pileggi, "PRIMA: passive reduced-order interconnect macromodeling algorithm," IEEE Transactions on Computer-Aided Design of Integrated Circuits and Systems, vol. 17, no. 8, pp. 645 -654, Aug. 1998.
[6] L. Knockaert and D. De Zutter, "Laguerre-SVD reducedorder modeling," IEEE Transactions on Microwave Theory and Techniques, vol. 48, no. 9, pp. 1469 -1475, Sept. 2000.

[7] P. K. Gunupudi, R. Khazaka, M. Nakhla, T. Smy, and D. Celo, "Passive parameterized time-domain macromodels for high-speed transmission-line networks," IEEE Transactions on Microwave Theory and Techniques, vol. 51, no. 12, pp. 2347 - 2354, Dec. 2003.

[8] L. Daniel, O. C. Siong, L. Chay, K. H. Lee, and J. White, "A multiparameter moment-matching modelreduction approach for generating geometrically parameterized interconnect performance models," IEEE Transactions on Computer-Aided Design of Integrated Circuits and Systems, vol. 23, no. 5, pp. 678 - 693, May 2004.

[9] F. Ferranti, G. Antonini, T. Dhaene, and L. Knockaert, "Guaranteed passive parameterized model order reduction of the partial element equivalent circuit (PEEC) method," IEEE Transactions on Electromagnetic Compatibility, vol. 52, no. 4, pp. 974-984, Nov. 2010.

[10] F. Ferranti, G. Antonini, T. Dhaene, L. Knockaert, and A. Ruehli, "Physics-based passivity-preserving parameterized model order reduction for PEEC circuit analysis," IEEE Transactions on Components, Packaging and Manufacturing Technology, vol. 1, no. 3, pp. 399 -409, Mar. 2011.

[11] H. Panzer, J. Mohring, R. Eid, and B. Lohmann, "Parametric model order reduction by matrix interpolation," $\mathrm{Au}$ tomatisierungstechnik, pp. 475-484, Aug. 2010.

[12] G. H. Golub and C. F. V. Loan, Matrix Computation. Maryland: Johns Hopkins University Press, 1996.

[13] E. W. Cheney, "Multivariate approximation theory : Selected topics," CBMS NSF Regional Conference Series in Applied Mathematics, vol. 51, Philadelphia, PA : SIAM, Dec. 1986.

[14] D. F. Watson, "Computing the n-dimensional delaunay tessellation with application to voronoi polytopes," The Computer Journal, vol. 24, no. 2, pp. 167-172, Feb. 1981.

[15] A. Weiser and S. E. Zarantonello, "A note on piecewise linear and multilinear table interpolation in many dimensions," Mathematics of Computation, vol. 50, no. 181, pp. 189 - 196, Jan. 1988.

[16] R. Rohrer and H. Nosrati, "Passivity considerations in stability studies of numerical integration algorithms," International Journal of Control, vol. 28, no. 9, pp. 857 - 866, Sept. 1981.

[17] R. W. Freund, "Krylov-subspace methods for reducedorder modeling in circuit simulation," J. Comput. Appl. Math., vol. 123, pp. 395-421, Nov. 2000. 